\title{
SOCIOLOGIE DES RELIGIONS
}

\section{Bibliographie sélective}

Publiée à l'occasion du cycle de conférences consacré à la Sociologie des religions en France, du 14 janvier au 2 juin 2020, cette bibliographie propose une sélection d'ouvrages et de documents disponibles en salle $\mathbf{J}$ de la Bibliothèque d'étude (Haut-de-jardin). A l'image du cycle de conférences qui l'inspire, cette bibliographie cherche à dresser un état des lieux de la réflexion sociologique sur la religion, en privilégiant les ouvrages récents, sans mettre spécialement en avant le phénomène de la radicalité religieuse, souvent privilégié par l'actualité éditoriale et médiatique.

Dès ses origines, la sociologie s'est intéressée au fait religieux : parmi les premiers ouvrages des pères fondateurs de la discipline figurent des études sur les pratiques religieuses, et des analyses fondamentales sur le phénomène de la croyance. Mais la sociologie des religions s'est beaucoup renouvelée depuis une trentaine d'années, tenant compte de mutations culturelles profondes qui n'ont pas épargné les croyances et les pratiques religieuses. Au-delà de l'observation des usages (appartenance déclarée à une religion, fréquentation des lieux de culte, visibilité dans l'espace social) la sociologie française récente s'est moins intéressée aux croyances en général qu'aux croyants en particulier, à leurs parcours et à leur manière de vivre leur foi, à une époque où les institutions en général, et religieuses en particulier, ne déterminent plus entièrement les croyances des individus.

\section{Pour commencer...}

Bobineau, Olivier et Tank-Storper, Sébastien

Sociologie des religions. Paris, Armand Colin, 2012. 126 p.

Salle J - Sociologie - [306.603 BOBI s]

Hervieu-Léger et Willaime, Jean-Paul

Sociologies et religion : approches classiques. Paris, Presses universitaires de France, 2001. 289 p. (Sociologies d'aujourd'hui)

Salle J - Sociologie - [306.6 SOCI 1]

Tarot, Camille

L'actualité de la religion : introduction critique aux sciences sociales des religions. Latresne, Le Bord de l'eau, 2019. $665 \mathrm{p}$.

Salle J - Sociologie - [306.6 TARO a]

Trigano, Shmuel

Qu'est-ce que la religion? La transcendance des sociologues. Paris, Flammarion, 2001. 331 p.

Salle J - Sociologie - [306.6 TRIG q]

Willaime, Jean-Paul

Sociologie des religions. Paris, Presses universitaires de France, 2012. 127 p. (Que sais-je ?)

Salle J - Sociologie - [306.6 WILL s]

\section{Ouvrages généraux}

Azia, Régine et Hervieu-Léger, Danièle (dir.)

[avec la collaboration de Dominique logna-Prat]

Dictionnaire des faits religieux. Paris, PUF, 2019. 1439 p. (Quadrige)

Salle J - Religion - [200.3 AZRI d] 
Béraud, Céline, Duriez, Bruno et de Gasquet, Béatrice de (dir.)

Sociologues en quête de religion. Rennes, Presses universitaires de Rennes, 2018. 237 p. (Sciences des religions)

Salle J - Sociologie - [306.6 BERA s]

Cholvy, Gérard et Hilaire, Yves-Marie

Le fait religieux aujourd'hui en France : les trente dernières années, 1974-2004. Paris, Ed. du Cerf, 2004.412 p. (Histoire)

Salle J - Sociologie - [306.609 44 CHOL f]

Dianteill, Erwan et Löwy, Michael

Sociologies et religion: II, Approches dissidentes. Paris, Presses universitaires de France, 2005.188 p. (Sociologies d'aujourd'hui)

Salle J - Sociologie - [306.6 SOCI 2]

Dianteill, Erwan et Löwy, Michael

Sociologies et religion: III, Approches insolites. Paris, Presses universitaires de France, 2009. 175 p. (Sociologies d'aujourd'hui)

Salle J - Sociologie - [306.6 SOCI 3]

Durkheim, Émile

Les formes élémentaires de la vie religieuse, édition critique par Myron Achimastos. Paris, Classiques Garnier, 2015. 825 p. (Bibliothèque des sciences sociales)

Salle J - Sociologie - [301.01 DURK o1]

Hervieu-Léger, Danièle

La religion en mouvement : le pèlerin et le converti. Paris, Flammarion, 1999. 289 p.

Salle J - Sociologie - [306.6 HERV r]

Kepel, Gilles

La revanche de Dieu : chrétiens, juifs et musulmans à la reconquête du monde. Paris, Ed. du Seuil, 1992.282 p.

(Points)

Salle J - Sociologie - [306.6 KEPE r]

Lassave, Pierre

La sociologie des religions : une communauté de savoir. Paris, EHESS, 2019. 408 p. (En temps \& lieux)

Salle J - Sociologie - [306.6 LASS s]

Lenoir, Frédéric

Les métamorphoses de Dieu : la nouvelle spiritualité occidentale. Paris, Plon, 2019. 512 p. (L'Abeille)

Salle J - Sociologie - [306.6 LENO m]

Liogier, Raphaël

Souci de soi, conscience du monde : vers une religion globale. Paris, A. Colin, 2012. 279 p.

Salle J - Sociologie - [306.6 LIOG s]

Piette, Albert

La religion de près : l'activité religieuse en train de se faire. Paris, Métailié, 1999. 271 p. (Leçons de choses)

Salle J - Anthropologie, ethnologie - [306.089 41 PIET r]

Simmel, Georg

La religion, trad. de l'allemand par Philippe Ivernel ; postf. de Patrick Watier. Belfort, Circé, 1998. 180 p. 
Salle J - Sociologie - [301.01 SIMM r]

Tarot, Camille

Le symbolique et le sacré. Paris, Éd. la Découverte, 2008. 910 p. (Textes à l'appui. Série Bibliothèque du MAUSS)

Salle J - Religion - [291 TARO s]

Weber, Max

Sociologie des religions, textes réunis, traduits et présentés par Jean-Pierre Grossein ; introd. de Jean-Claude Passeron. Paris, Galimard, 1996. 545 p.

Salle J - Sociologie - [301.01 WEBE s]

\section{Ouvrages et contributions thématiques}

Altglas, Véronique

«Exotisme religieux et bricolage ». Archives de sciences sociales des religions [En ligne], 2014, 167

Disponible sur : http://journals.openedition.org/assr/26229

Altglas, Véronique

Le nouvel hindouisme occidental. Paris, CNRS, 2005. 227 p. (CNRS sociologie)

Salle J - Sociologie - [306.694 ALTG n]

Altglas, Véronique

« «Les mots brûlent»: Sociologie des nouveaux mouvements religieux et déontologie ». Archives de Sciences Sociales des Religions [en ligne], 2005, 131-132

Disponible sur : http://journals.openedition.org/assr/3264

Aubin-Boltanski, Emma, Lamine, Anne-Sophie, Luca, Nathalie (dir.)

Croire en actes : distance, intensité ou excès [actes du colloque, Paris, École des hautes études en sciences sociales, 7-8 février 2011]. Paris, l'Harmattan, 2014. 224 p. (Religions en questions)

Salle J - Sociologie - [306.6 AUBI c]

Bastian, Jean-Pierre

Pluralisation religieuse et logique de marché. Bern, Peter Lang, 2007. 216 p.

Salle J - Sociologie - [306.6 BAST p]

Baubérot, Jean

La laïcité falsifiée. Paris, la Découverte, 2011. 212 p. (Cahiers libres)

Salle J - Sociologie - [306.609 44 BAUB 1]

Béraud, Céline

Le métier de prêtre : approche sociologique. Paris, les Éd. de l'Atelier, 2006. 157 p.

Salle J - Sociologie - [306.682 BERA m]

Béraud, Céline, Guguelot, Frédéric et Saint-Martin, Isabelle (dir.)

Catholicisme en tensions. Paris, EHESS, 2012. 326 p. (En temps \& lieux)

Salle J - Religion - [200.1 BERA c]

Béraud, Céline et Portier, Philippe

Métamorphoses catholiques : acteurs, enjeux et mobilisation depuis le mariage pour tous. Paris, Editions de la Maison des sciences de l'homme, 2015. 205 p. (Interventions)

Salle J - Sociologie - [306.682 BERA m] 
Béraud, Céline, Galembert, Claire et Rostaing, Corinne

De la religion en prison. $\mathrm{R}, 2016.358 \mathrm{p}$.

Salle J - Sociologie - [306.609 44 BERA d]

Bertina, Ludovic, Carnac, Romain et Fauches, Aurélien (et al.)

Nature et religions. Paris, CNRS, 2013. 220 p.

Salle J - Anthropologie, ethnologie - [306.6 NATU]

Carnac, Romain, Guidi, Diletta et Roucoux, Guillaume

Les autorités religieuses face aux questions de genre : reconfigurations contemporaines des mondes confessionnels autour des nouvelles questions sexuelles. Rennes, Presses universitaires de Rennes, 2019. 186 p.

Salle J - Religion - [200.04 CARN a]

Bethmont, Rémy et Gross, Martine

[avec les contributions de Rebecca T. Alpert, Céline Béraud, Rémy Bethmont et al.]

Homosexualité et traditions monothéistes : vers la fin d'un antagonisme. Paris, Labor et fides, 2017. 387 p.

Salle J - Religion - [200.04 BETH h]

Dianteill, Erwan, Hervieu-Léger, Danièle et Martin, Isabelle (dir.)

La modernité rituelle: rites politiques et religieux des sociétés modernes. Paris, L'Harmattan, 2009. 204 p. (Religions en questions)

Salle J - Sociologie - [306.4 DIAN m]

Dumons, Bruno et Gugelot, Frédéric (dir.)

Catholicisme et identité: regards croisés sur le catholicisme français contemporain, 1980-2017. Paris, Karthala, 2017. 331 p. (Signes des temps)

Salle J - Religion - [270.944 DUMO c]

Endelstein, Lucine, Fath, Sébastien et Mathieu, Séverine (dir.)

Dieu change en ville : religion, espace, immigration. Paris, L'Harmattan, 2000. 220 p. (Religions en questions)

Salle J - Sociologie - [306.6 ENDE d]

Fath, Sébastien

Du ghetto au réseau : le protestantisme évangélique en France, 1800-2005. Genève, Labor et Fides, 2005.425 p. (Histoire et société)

Salle J - Sociologie - [306.609.44 FATH d]

Gotman, Anne

Ce que la religion fait aux gens: sociologie des croyances intimes. Paris, Ed. de la Maison des sciences de l'homme, 2013. 289 p. (54)

Salle J - Sociologie - [306.6 GOTM c]

\section{Gotman, Anne}

«Comment la religion vient aux gens ». Archives de sciences sociales des religions [En ligne], 2013, 163

Disponible sur : http://journals.openedition.org/assr/25281

Gross, Martine, Mathieu, Séverine et Nizard, Sophie (dir.)

[avec les contributions de Joëlle Allouche-Benayoun, Laurent Barry, Céline Béraud et al.]

Sacrées familles ! : changements familiaux, changements religieux. Toulouse, Eres, 2011. 264 p.

Salle J - Sociologie - [306.8 GROS s]

Hervieu-Léger, Danièle 
Catholicisme, la fin d'un monde. Paris, Bayard, 2003. 334 p.

Magasin - [2003-114340]

Hervieu-Léger, Danièle

Vers un nouveau christianisme ? : introduction à la sociologie du christianisme occidental. Paris, Ed. du Cerf, 1987. 395 p. (Sciences humaines et religions)

Salle J - Sociologie - [306.6 HERV v]

Jonveaux, Isabelle

Dieu en ligne : expériences et pratiques religieuses sur Internet. Montrouge, Bayard, 2013. 251 p.

Salle J - Sociologie - [306.6 JONV d]

Le Bras, Gabriel

L'église et le village. Paris, Flammarion, 1976. 289 p. (Nouvelle bibliothèque scientifique)

Salle J - Sociologie - [306.601 LEBR e]

Lamine, Anne-Sophie

Identités religieuses et monde commun : penser les idéaux, les attachements et la participation sociale avec John Dewey. Paris, L'Harmattan, 2018. 229 p. (Logiques sociales)

Salle J - Sociologie - [306.6 LAMI q]

Lamine, Anne-Sophie (dir.)

Quand le religieux fait conflit: désaccords, négociations ou arrangements. Rennes, Presses universitaires de Rennes, 2014. 239 p. (Sciences des religions)

Salle J - Sociologie - [306.6 LAMI q]

Lamine, Anne-Sophie_et Sarg, Rachel

«La religion en prison». Archives de sciences sociales des religions [En ligne], 153 |janvier-mars 2011

Disponible sur : http://journals.openedition.org/assr/22761

Lamine, Anne-Sophie

«Les formes actuelles du retour du religieux ». Regards sur l'actualité. Paris, La Documentation française, $\mathrm{n}^{\circ} 364$ octobre 2010, pp. 21-32

Salle D - Périodiques - [GENE Rega actu]

Lamine, Anne-Sophie

«Les croyances religieuses des jeunes ». Dans Le Breton, David et Marcelli, Daniel (dir.). Dictionnaire de la jeunesse et de l'adolescence. Paris : Presses universitaires de France, 2010, pp. 714-718

Salle J - Psychologie, psychanalyse - [155.5 LEBR d]

Lasserre, Henri

Le phénomène des conversions religieuses : vers une reconstruction de soi ? Paris, Cognitia: ESF sciences humaines, 2016. $93 \mathrm{p}$.

Salle J - Sociologie - [306.609 44 LASS p]

Le Pape, Loïc

Une autre foi : itinéraires de conversions religieuses en France : juifs, chrétiens, musulmans. Aix-en-Provence, Presses universitaires de Provence, 2015. 198 p. (Sociétés contemporaines)

Salle J - Sociologie - [306.609 44 LEPA a]

Le Pape, Loïc.

« «Faire communauté ». Comment les Églises fabriquent leurs convertis? ». Théologiques [en ligne], volume 21, numéro 2, 2013.

Disponible sur : https://doi.org/10.7202/1028464ar 
Mathieu, Séverine

La transmission du judaïsme dans les couples mixtes. Ivry-sur-Seine, Ed. de l'Atelier, 2009. 174 p.

Salle J - Sociologie - [306.696 MATH t]

Mayer, Jean-François et Kranenborg, Reender (dir.)

La naissance des nouvelles religions. Genève, Georg, 2004. 212 p.

Salle J - Sociologie - [306.699 44 MAYE n]

Messner, Francis (dir.)

L'affiliation religieuse en Europe. Strasbourg, Presses universitaires de Strasbourg, 2017. 212 p. (Société, droit et religion)

Salle D - Droit - [342.408 MESS a]

Obadia, Lionel et Zwilling, Anne-Laure (dir.)

Minorité et communauté en religion. Strasbourg, Presses universitaires de Strasbourg, 2016. 293 p. (Collections de l'Université Robert Schuman. Société, droit et religion en Europe)

Salle J - Sociologie - [306.6 OBAD m]

Puzenat, Amélie

Conversions à l'islam : unions et séparations. Rennes : Presses universitaires de Rennes, 2015. 262 p. (Sciences des religions)

Salle J - Sociologie - [306.697 PUZE c]

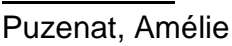

"Ritualités dans la (dis-)continuité : agencements et petits arrangements de femmes françaises converties à l'islam. » Théologiques [en ligne], volume 21, numéro 2, 2013

Disponible sur : https://doi.org/10.7202/1028467ar

Raison Du Cleuziou, Yann

Qui sont les cathos d'aujourd'hui ? : sociologie d'un monde divisé. Paris, Desclée de Brouwer, 2014.332 p. (Confrontations)

Salle J - Religion - [261 RAIS q]

Rochefort, Florence et Sanna, Maria Eleonora (dir.)

Normes religieuses et genre : mutations, résistances et reconfigurations, XIXe-XXIe siècle. Paris, Armand Colin, 2013 (recherches)

Salle J - Sociologie - [306.6 ROCH n]

\section{Roy, Olivier}

La sainte ignorance : le temps de la religion sans culture. Paris, Ed. du Seuil, 2008. 275 p. (La Couleur des idées) Salle J - Sociologie - [306.6 ROY s]

$\overline{\text { Tietze, Nikola }}$

Jeunes musulmans de France et d'Allemagne : les constructions subjectives de l'identité. Paris, l'Harmattan, 2008. 237 p. (Religion et sciences humaines)

Salle J - Sociologie - [306.609 4 TIET j]

Todd, Emmanuel

Qui est Charlie : sociologie d'une crise religieuse. Paris, Seuil, 2015. 242 p.

Salle J - Sociologie - [306.609 44 TODD q]

Tricou, Josselin et Stambolis-Ruhstorfer, Michael 
«La lutte contre la «théorie du genre » en France : pivot d'une mobilisation religieuse dans un pays sécularisé ». Dans Paternotte, David et Kuhar, Roman (dir.). Campagnes anti-genre en Europe. Des mobilisations contre l'égalité. Lyon : Presses universitaires de Lyon, 2018, p. 143-166

Salle J - Sociologie - [305.42 KUHA c]

Willaime, Jean-Paul

[entretiens avec A. - Martin Meunier]

La guerre des dieux n'aura pas lieu : itinéraire d'un sociologue des religions. Genève, Labor et fides, 2019. 360 p.

Salle J - Sociologie - [306.6 WILL g]

Zwilling, Anne-Laure (dir .)

Les minorités religieuses en France : panorama de la diversité contemporaine. Montrouge, Bayard, 2019. 1039 p. Salle J - Religion - [200.944 ZWIL m]

\section{Revues et publications périodiques}

Archives des sciences sociales des religions [en ligne]

Paris, CNRS, 1973-

Disponible sur : https://journals.openedition.org/assr/ (consulté le 18.12.2019)

Agenda pour une sociologie critique des religions [en ligne]

Paris, Groupe Sociétés, religions, laïcités, 2017-

Disponible sur : https://acsrel.hypotheses.org/ (consulté le 08.01.2019)

\section{Pour @ller plus loin}

\section{Sélection de sites Internet et de carnets de recherches}

Association française de sociologie

Sociologie \& religions [en ligne]. Disponible sur : https://socioreligions.wordpress.com/ (consulté le 18.12.2019)

Mayeur, Jean-François

Religioscope [en ligne]. Disponible sur : https://www.religion.info/ (consulté le 18.12.2019)

Zwilling, Anne-Laure

Sociorel: Actualités concernant la SOCIOlogie et les RELigions en Europe [en ligne]. Disponible sur: https://sociorel.hypotheses.org/ (consulté le 18.12.2019) 